\title{
Surgical Aspects of Rehabilitation
}

\author{
THE FOOT
}

'The following lesions may delay rehabilitation, and are amenable to modern treatment.

(a) PLANTAR ULcer. Damage to the neuropathic foot may occur superficially between skin and ground, or deep between bony skeleton and soft tissues as a result of pressure and shear stresses during walking.

The deep damage proceeds by necrosis (pre-ulcerative state) to subcutaneous blister and finally ulccration. Ulccration can be avoided by early recognition and treatment, but if it threatens or has recently occurred, healing can be obtained by the avoidance of the stresses of walking. The simplest is bed-rest with elevation of the foot; but rigidsoled footwear, firmly attached to the foot, is as effective. So also is a walking plaster cast, and uncomplicated ulcers will heal within six weeks. Bone involvement may also delay healing, but will not prevent it if immobilisation is prolonged. Removal of sequestra may accelerate healing, but operative interference should not be attempted except by experienced surgcons, and should aim at producing a plantigrade foot. If the deformity is too severe to obtain this, amputation may be needed in preparation for an artificial limb (simple plastic prostheses are becoming available).

Recurrence of plantar ulcer is avoided by footwear and a simple soft insole is adequate in many cases; more severe ones need footwear with 
the following criteria: rigid sole, soft insole (microcellular rubber is degrees 'Shore'), a rocker or rocker-shaped sole, and firm strapping of heel to footwear. A moulded shoe may be useful for severe deformity, and walking should be limited. Education of the patient is an essential part of treatment.

(b) Neuropathic bone and joint. Bone and joint damage follows repeated microtraumata at any anesthetic joint of the foot; it often accompanies, but is not caused by, plantar uleer. 'The lesion should be suspected whenever a painless swelling is present and X-ray should be done. Treatment of the neuropathic joint is essentially the achievement of undeformed stability by surgical or non-surgical methods.

(c) DROP-FOo'T. Paralysis of dorsiflexion and eversion of the foot follow's latual popliteal neuritis. In acute cases, immediate plaster immobilisation, including the knee in slight flexion, may succeed in preventing permanent palsy. Failing this, the effect of gravity rapidly produces permanent foot-drops in eversion. Permanent foot-drops if mobile, should be corrected to prevent toe and plantar damage; footwear with foot-drop stop, or surgical transplant of tibialis posterior is used. I.ong standing foot-drops, with bony changes and joint-contractures, demands corrective arthrodesis of the mid-tarsus with lengthening of tende achilles.

(d) CLAW-TOEs. Cilaw-toes result from posterior tibial neuritis; they expose toe-tips and the sole to possible damage, and make shoe-fitting difficult. 'The deformity can be corrected by tendon transplant or by arthrodesis at the interphalangeal joint. Amputation single toes is useful, but removal of the big toe should be avoided.

(e) infective VAsGular Lesions. Infection of blood and lymph vessels of the foot and lower-leg is common during repeated and uncontrolled septic episodes of the foot. Intractable conditions of post-phlebitic syndrome or of chronic lymphoedema may result. Early recognition and treatment is important; regular hygiene of the foot, with compressive bandages, will arrest the development of the developed lesions.

CONGLusion. With modern treatment, no complication of leprosy in the foot should hinder the rehabilitation of a patient recovering from the disease.

\section{DEFORMITIES OF THE FACE}

All the deformitics of the face are amenable to correction by surgery. As visible stigma of leprosy, their correction is important in the general problem of rehabilitation for the lay person often associates deformity with active disease.

The operative treatment for deformities of the face is based on the principles and techniques of plastic surgery. Any doctor undertaking this type of surgery must be adequately trained in this speciality if satisfactory results are to be achieved. 
The deformities of the face in leprosy comprise of:

(a) The depressed nose.

(b) Loss of eycbrows.

(c) Deformity of th ears.

(d) Wrinkling of the facial skin.

(c) Lagophthalmos.

Except lagophthalmos, all these deformities are the result of lepromatous disease and correction should be preferably deferred till the disease is arrested.

Nose. This is the most prominent stigma of this discase. 'The saddle nose deformity is the result of primary ulceration of the mucous membrane followed by exposure necrosis of the underlying cartilage and bony framework. In general the skin, though infiltrated, is not ulcerated though this is possible in some cases.

It was formerly the general practice to encise the remains of the nose and reconstruct a new nose by a forchead skin flap. 'This is not necessary in th majority of cases where the skin and tip of the nose are present intact though displaced. 'The missing lining is replaced by a forehead flap of a free skin graft introduced into the cavity behind the nose which is produced by freeing the skin from its anchorage to the underlying bone. 'The skin graft is carried on a mould which is later replaced by a dental prothesis or if the patient desires permanent support may be given by a bone graft.

In minor nasal depression an implant of cartilage or bone may be undertaken without a preliminary skin graft. Inner implants may also be employed.

In the rare cases of total destruction of the nose, a forehead rhinoplasty is undertaken.

ЕҮЕВR@Ws. 'The correction of this deformity is of psychological importance. 'This may be done by grafting hair bearing scalp as a free graft or as a pedicle graft.

EARs. 'The deformity may be in the form of an elongated lobule or an irregular destruction of the pinna of the car. This can be readily corrected by suitable incisions.

FACIAL WRINKLES. 'This is the result of loss of normal skin elasticity and produces an appearance of premature ageing. It is corrected by incision of the redundant skin.

Gynaecomastia is a common deformity and may be associated with pain. This is corrected by removing the excess fat and breast tissue through an incision in the margin of the areola.

In leprosy, the common defects of claw-hand and paralysed thumb are often complicated by contracture, lepra reaction and absorption of the 
phalanges. It should be emphasised that it is useless to attempt most surgical reconstruction before the hand has been fully mobilised, and it is equally important post-operatively to train the patient in re-education of his transferred muscles if anything like satisfactory results are desired. Moreocver, because of severe disasters that can follow ill-advised surgery or post-operative sepsis it is strongly advised that such reconstructive surgery should only be done where there is a surgeon trained in this work, and also where there is provision for adequate physiotherapy.

CLAW-HAND. Where there is pre-operative mobility with intact tendons (i.e., where there is no dorsal expansion damage), the best treatment for claw-hand is to provide a new motor for the paralysed intrinsic muscles.

Those commonly used are:

(a) Extensor carpi radialis longus with a frec graft.

(b) Flexor digitorum sublimis from one linger.

(c) Extensor indicis and extensor digiti minimi.

Capsuloplasty is also sometimes practised.

For those cases in which passive mobility is unobtainable pre-operatively so that passive extension of the proximal interphalangeal joints is limited by 45 degrees or more, it is usually advisable to arthrodese the interphalangeal joints at approximately 2.5 degrees short of full extension.

THE THUMB. 'The commonest complication of paralysis in the thumb is thumb web contracture. If the thumb cannot be brought into full opposition passively, it is necessary to do some form of web-plasty before providing a motor tendon to the thumb. A web-plasty with a full thickness skin graft on the dorsum, and sometimes a Z-plasty can be used.

An accepted operation for correction of the paralysis is transfer of the flexor digitorum sublimis from the $4^{\text {th }}$ finger, re-routed from a position near the pisiform or distal to the transverse carpal ligament, in two slips to the thumb, one into the extensor over the proximal phalanx and the other into the adductor insertion. Difficulty in extension of the terminal phalanx is common in ulnar - median paralysis which readily provokes a chronic flexion of the phalanx. If this disability is not corrected even after tendon transfer or if there is post-operative instability of the metacarpo-phalangeal joint, then arthrodesis of the MP joint is advisable. Interphalangeal arthrodesis of the thumb is only used in cases in which th are is fixed flexion of the joint. Where the lesion is purcly ulnar, the sublimis from the $4^{\text {th }}$ finger may be re-routed directly from the centre of the palm as a single slip to be inserted into the dorsal expansion over the proximal phalan of the thumb. Another method that has been used is that of passing the extensor indicis proprius through the 2nd interosseous space to the mid-palm and thus to the thumb.

RADIAI. PALSY. Radial palsy is uncommon and occurs only with ulnar and low median palsy. Although arthrodesis of the wrist was recommended in the lokyo report, the panel now recommends muscle transfers as used in poliomyelitis. 
WAstring; in THE THUMB WhB. 'The unsightly wasting in the thumb web hollow can be filled with dermis grafts or IVíson for cosmetic purposes.

AIDAN(EI) ABSORPTION OF THE FIN(GERS ANI) THE THUMB. EVCn where there is severe absorption of the fingers the hand may still be useful if the thumb is good. Where there is advanced absorption of the thumb, a dece) web-plasty can provide further function.

It is disappointing to attempt to lengthen the fingers with bone grafts or pedicles.

In very advanced absorption and where it is technically possible, consideration mat be given to use of the artificial articulated hand.

IS'T RESOIUTH ON OF THE PANEL,

This Congress is gravely concerned that under the very eyes of doctors and para-medical workers in many anti-leprosy campaigns deformity and blindness are being allowed to develop, which could be prevented by simple advice and inexpensive treatment. 'The development of such deformities not only makes the ultimate rehabilitation of such cases very difficult, but causes lack of confidence and co-operation of patients in the medical treatment.

This Congress therefore resolves that in cvery anti-leprosy campaign the doctors and para-medical workers should be trained to look for danger signs in hands and feet and eyes, and should give advice and simple treatment to prevent deformity and blindness. The ratio of patients to workers should not become so high as to make this impossible.

\section{NI) RESOLUTION OF THE PANEL}

Whereas in many countries where leprosy is common there are no rehabilitation scrvices and no trained personnel, and whereas the governmental anti-leprosy programmes are unable to develop adequate rehabilitation services, this Congress

Resolves to call upon international and voluntary rchabilitation agencies to establish pilot projects for reconstructive surgery and physical rehabilitation at strategic centres, preferably in association with medical schools, to which staff from any country could be sent for training.

This Congress suggests that the United Nations Rehabilitation Agency and the International Society for the Rehabilitation of the Disabled should call a group of voluntary socicties together to consider means of implementing this resolution. 